\title{
Bend Sensor Based on Fibreoptics and Concept for a Compact Evaluation Unit
}

\author{
S. Kibben ${ }^{1} *$ M. Kropp ${ }^{2}$, G. Dumstorff ${ }^{2}$, M. Koerdt ${ }^{1}$, W. Lang ${ }^{2}$ and F. Vollertsen ${ }^{1}$ \\ ${ }^{1}$ BIAS - Bremer Institut für angewandte Strahltechnik GmbH, Klagenfurter Str. 2, 28359 Bremen, Germany \\ ${ }^{2}$ IMSAS - Institute for Microsensors, -actuators and -systems, Otto-Hahn-Allee, Building NW1, 28359 Bremen, Germany \\ *E-Mail: kibben@bias.de
}

Summary: A concept study for a bend sensor based on a fibre Bragg grating (FBG) and a compact evaluation unit for the investigated type of sensor is presented. The fibreoptic sensor is capable of resolving the radius and the direction of the bend. The measurement data evaluation unit is capable of resolving multiple distributed FBG sensors, which are present in a single fibre at different positions.

Keywords: Optical, Bend, Sensor.

\section{Introduction}

Fibre-optical sensors based on Bragg gratings are experiencing a breakthrough since the discovery of a simple way to inscribe the periodical perturbation of the refractive index into the light-guiding core of the fibre [1]. First, these FBG were used in the field of fibreoptic telecommunication, where the narrowband filters were used as wavelength-selective reflectors building the basis of several fibre optical components. However the sensitivity to changes of the temperature and the strain of the reflected wavelength already was recognized by Kenneth $\mathrm{O}$. Hill and his colleagues, who recorded the first fibre Bragg grating [2].

Morey, Meltz and Glenn later characterized the sensitivity of the reflected wavelength to applied strain [3]. The sensitivity of the Bragg wavelength to temperature and strain is related to changes in the effective refractive index and to changes in the grating period. Both of them are affected by changes of temperature and strain. A change in temperature mainly changes the effective refractive index, as the thermal expansion coefficient of the used quartz glass is very small $\left(\sim 0.5 \cdot 10^{-6} / \mathrm{K}\right)$. Strain affects the grating period and due to the linear expansion also the refractive index is changed, which can be expressed by the photoelasic coefficient of the isotropic material. Later, a third sensitivity was described, which also affects the effective refractive index [4]. Here the cladding material is removed in the area of the FBG, so that changes in the refractive index of the surrounding media affect the guided light via the evanescent field.

Since then, every sensing principle utilizing FBG has been a conversion of the intended measurand into strain, temperature or changes of refractive index in the evanescent field.

The first FBG bend sensor relied on a simple principle involving two FBG fixed on a cantilever. When the cantilever was bent, the Bragg wavelengths of both FBG shift [5]. With this setup it is possible to resolve the strain at both sides of the cantilever thus allowing to resolve the bending radius in one direction. The measurement is independent of the ambient temperature. However, this concept does not allow resolving bending which does not occur in the intended direction. The sensor also cannot easily be integrated into lightweight structures like fibre reinforced plastics, as the materials properties are massively disturbed by the glass fibre.

Other concepts which are more adapted for the integration into thin lightweight composite structures are based on a two or multiple core optical fibre, in which a bending has different effects on each core. This also allows resolving the actual direction of the bending [6], but the coupling to such fibres is not trivial and also the analysis of the optical signals is complicated and often cost-intensive equipment is necessary.

For analysing an optical signal of an FBG many concepts exist. Simple concepts are based on two edge-filters with an overlap in their transmission spectra. Using a photodiode behind both filters the ratio is a measure for the wavelength of the incoming light. This system does not allow for multiplexing, as two separated wavelengths are interpreted as a single signal.

A different scheme consists of a grating, which separates the incoming wavelengths by means of diffraction. The signal is directed onto a line array of a charge coupled device (CCD). This measurement principle is relatively fast but is not feasible for FBG reflecting in telecommunications wavelength area, as silicon-based $\mathrm{CCD}$ are only sensitive in the visible up to a wavelength of $1.1 \mu \mathrm{m}$.

In a third scheme, a tuneable infrared (IR) laser diode is used to scan the whole wavelength range. For each adjusted wavelength, the reflected power is recorded. These schemes offer a high precision, but have deficits in their readout speed and are costly.

The aim of this study is to develop a bending sensor based on a FBG and a compact evaluation unit for readout of the optical signals of the FBG. The developed sensor principle is capable of resolving the radius of curvature as well as the direction of bending. The evaluation unit is a simple approach to enable the readout of any FBG based sensor in a scanning principle.

\section{Approach}

\subsection{Fibre Bragg grating based bend sensor}

The concept of the bending sensor resolving the radius of curvature and the direction of the bending bases on two different principles of affecting the guided light, which passes the bent optical fibre and is reflected by the FBG inside the fibre core.

The first effect is well-known. When the fibre is bent, the inner part is being compressed and the outer part is being stretched. In classical optical fibres the core is in the centre of the cladding, thus lying in the neutral axis of the circular fibre. When the core is not placed in the neutral axis, it is exposed to strain when the fibre is 
bent. The strain is noticeable as a shift of the Bragg wavelength $\lambda_{\mathrm{B}}$. This also happens when a round optical fibre is side-polished to a Dshape. A D-shaped fibre or a fibre with eccentrical core has one axis of symmetry, what allows the resolution of the bending in a range of 180 degrees.

The second effect affects the two stages of polarization in the fibre. When the fibre is lying straight, both polarizations have the same effective refractive index. When the fibre is bent, birefringence occurs [7]. This effect is so small, that it cannot be recognized on the Bragg wavelength with standard equipment. The birefringence in the bent area of the fibre results in a separation of the electrical field maxima of the two states of polarization. Thus, different amounts of the field are guided inside the core of the fibre. As a result the reflectance of a FBG differs for both polarizations, as the reflection only takes place inside the core. This effect also depends on the grating parameters. A highly reflecting FBG will not show significant changes in the reflectance of both polarizations when it is bent.

On a classical optical fibre the difference will not be measurable unless the fibre is analysed using polarized light in different orientations. When the FBG is inscribed into a fibre with a high intrinsic birefringence, there is one reflection maximum for both perpendicular states of polarization. Depending on the orientation of the fibre, the bend affects both states of polarization. The effect can be described with a cosine dependence with two minima and maxima over the 360 degree allowing a resolution of the applied bending over 90 degree.

\subsection{Evaluation unit for $F B G$ readout}

The suggested readout principle is a scanning one, but here the scanning device is not the source (i.e. IR laser diode) but a filter. The input of the filter is the reflected signal of the FBG. The filter itself is a fibre-coupled Fabry-Perot interferometer (FPI). These devices are very small and suitable for the installation on lightweight structures i.e. in the field of transportation. The fibre-coupled FPI can be scanned via an applied voltage. The devices are commercially available with a high finesse. This offers the possibility to combine several FBG in a single fibre (i.e. offering distributed measurements with a single fibre integrated into a structure). Behind the FPI the intensity at the adjusted wavelength is read out via a photo detector. In the case of the here investigated bend sensitive FBG each polarization has to be read out separately. This can be done via a well-placed birefringent crystal.

\section{Experimental set-ups}

\subsection{FBG inscription}

The FBG were inscribed into commercially available photosensitive optical fibres using a krypton fluoride excimer laser (Lambda Physik LPX 305i) emitting at $248 \mathrm{~nm}$. The laser has a spatial coherence of about $0.5 \mathrm{~mm}$. The laser beam profile was reduced to $1 \mathrm{~cm} \times 1 \mathrm{~cm}$ via an aperture. The beam was directed onto a phase mask optimized for $248 \mathrm{~nm}$, which produces a nearly perfect sinusoidal interference pattern. The pulse fluence of the laser was set to $150 \mathrm{~mJ} / \mathrm{cm}^{2}$ and slightly varied over the inscription process. $10 \%$ of the pulse energy was directed onto a calibrated detector in order to control the inscription via the cumulative fluence. Each grating was recorded with a cumulative fluence of $1 \mathrm{~kJ} / \mathrm{cm}^{2}$.

Gratings were inscribed into two different optical fibres (GF1, PS-PM980, both produced by Nufern). The GF1 fibre is optimized for being spliced to standard telecommunications fibre (i.e. Corning
SMF-28). The PS-PM980 fibre is a high birefringence fibre preserving two reflection maxima for one inscribed FBG. Gratings recorded in the GF1 fibre are labelled GF. FBG recorded in the PSPM980 fibre are labelled PM. When the grating was side-polished after the recording, it will have an attached D (D-shaped) in the labelling. When the fibres shape was untouched, it will have an attached C (circular).

\subsection{Test bench}

For reproducible bending of the FBG a test bench consisting of two rotators with a fibre chuck in the rotation axis was employed. The rotators were placed with a right angle between their rotation axes; the distance of them could be adjusted. Via the length of the fibre and the distance of the two rotators the bending radius could be adjusted. The effective bending direction of the fibre could be altered by turning the two rotators in the same direction.

\subsection{Laboratory readout setup}

For reading out the FBG in the laboratory, a broadband source mainly emitting in the C-band was selected. The light was coupled into a circulator, where it then was directed into the fiber with the inscribed FBG. The reflected light again was directed into the circulator, from where it was passed to an optical spectrum analyser (OSA). The main parameters recorded with the OSA were the Bragg wavelength, the intensity at this wavelength and the $3-\mathrm{dB}$ bandwidth of the FBG.

\section{Results}

\subsection{FBG bending}

Four different FBG were investigated. Two FBG were recorded in each type of fibre. One of them was side-polished (sample GF-D and PM-D) and the other one was kept untouched (samples GF-C and PM-C). The spectra of the FBG were investigated under bending radii of $25 \mathrm{~mm}, 50 \mathrm{~mm}$ and $100 \mathrm{~mm}$.

When the sample GF-C was investigated, the intensity of the Bragg signal did not show any major tendency or change. The Bragg wavelength varied very slightly over the bending radius. The variation increased with decreasing radius. The variation showed one minimum and one maximum. The sample GF-D showed a strong shift of the Bragg wavelength over the bending angle, which also increased with reduced bending radius (see figure 1).

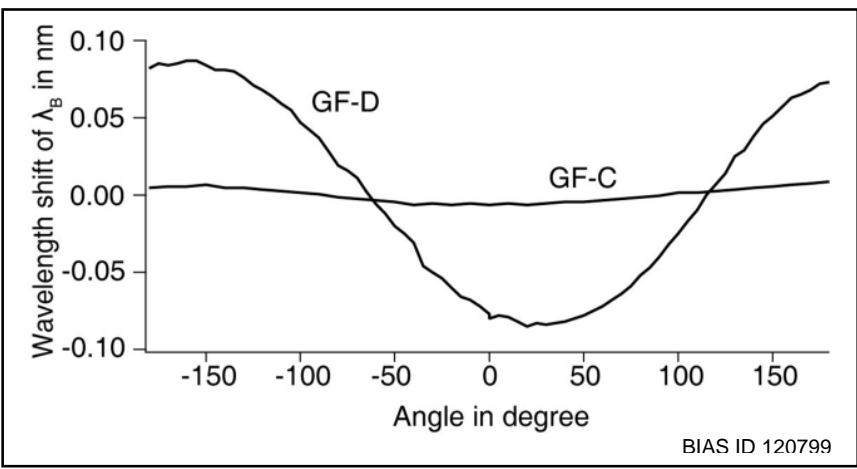

Figure 1. Wavelength shift of the sample GF-D over the bending angle. The bending radius is $25 \mathrm{~mm}$, the polishment of GFD has a depth of $10 \mu \mathrm{m}$. 
The sample PM-C (incorporating two reflection maxima, one for each state of polarization) did not show a significant wavelength shift. The intensity of both Bragg maxima changed over the bending angle showing two maxima and minima. When one state of polarization had its maximum, the other was at a minimum and vice versa (see figure 2).

When the sample PM-D was investigated, the Bragg wavelength of both Bragg maxima showed a major shift over the bending angle, as already observed on the sample GF-D. Again, the strength of the shift depended on the bending radius. The recorded intensity of the Bragg maxima did not follow any analytically expressible tendency (see figure 3). Several local maxima and minima were detectable over the bending angle. These also changed over the bending radius.

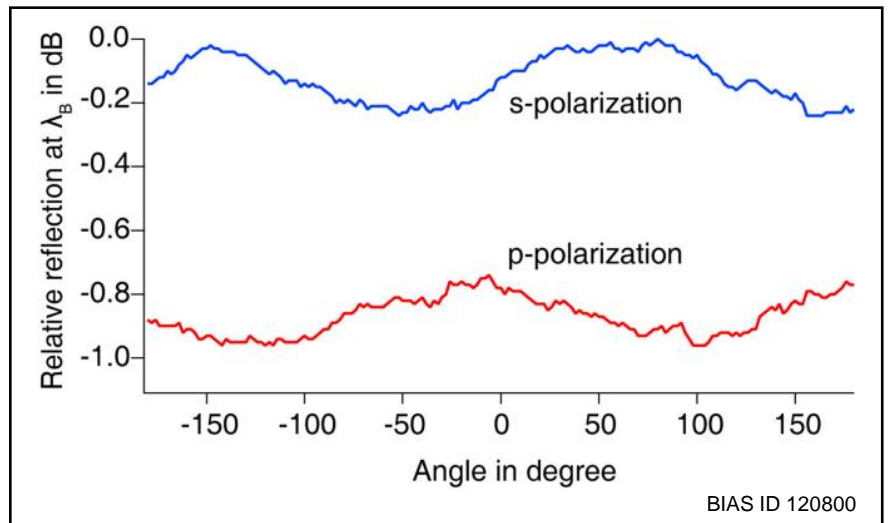

Figure 2. Intensity change of the sample PM-C over the bending angle. The bending radius is $50 \mathrm{~mm}$.

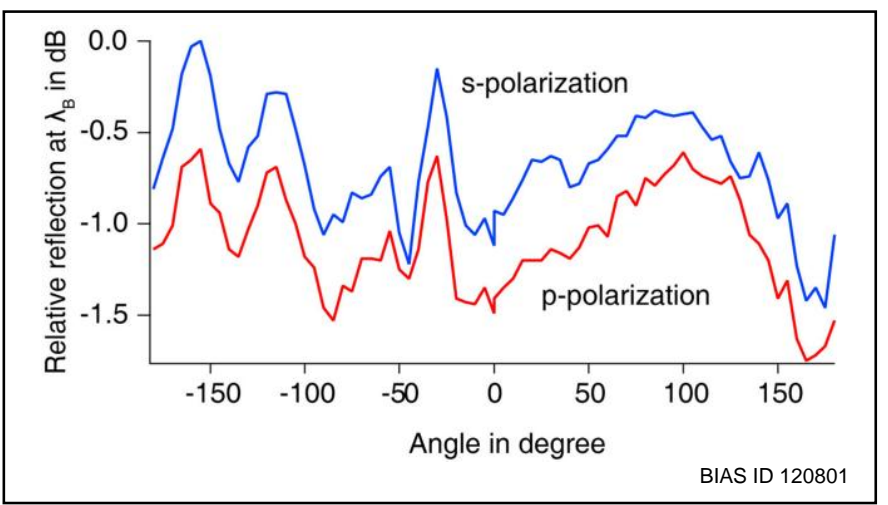

Figure 3. Intensity change of the sample PM-D over the bending angle. The bending radius is $50 \mathrm{~mm}$.

\subsection{FPI characterization}

For a correct interpretation of the data recorded via a setup consisting of an FPI and a photo detector, several characteristics have to be investigated. The finesse (the ratio of the transmission bandwidth and the free spectral range) describes the utilizable wavelength range for Bragg signals to be measured and the transmission bandwidth, which describes the minimum resolution of the measurement.

The transmission band was characterized with a tuneable laser source ( $1 \mathrm{pm}$ resolution) and a fibre-coupled photo detector. The FPI had a transmission bandwidth of $10 \mathrm{pm}$. The free spectral range was measured with a broadband source and an OSA; it was $0.1 \mu \mathrm{m}$ at a wavelength of $1.5 \mu \mathrm{m}$.

The transmission wavelength could be adjusted via an applied voltage. The wavelength shift in dependence of the applied voltage was characterized using a broadband source and an OSA. The wavelength shift depended linearly on the applied voltage. The measurement was repeated for several times and it was found out that the transmission wavelength was not perfectly stable.

\section{Conclusion}

It was shown that bending can be resolved utilizing special FBG. The angle and the radius can be resolved within certain periods of 180 and 90 degree respectively depending on the used principle. The results suggest a full resolution on the bending angle and the direction of the bending when both principles are combined.

Here, the combination of both principles delivered a resolution of 180 degree. The combination did not deliver the expected results as the polarization maintaining fibre was lossy and the leaky mode coupled back into the fibres light-guiding core, hence overlaying the measurable effects. It is assumed that in a polarization-maintaining fibre which is designed to work in the wavelength range of $1.5 \mu \mathrm{m}$ the losses will not overlay the observed effects of intensity change.

A concept study for a space-saving evaluation unit for reading out the investigated FBG which can be installed in transportation units such as aircrafts was presented. It allows reading out fibres with distributed FBG with different Bragg wavelengths with a resolution of $10 \mathrm{pm}$.

\section{Acknowledgement}

The authors gratefully acknowledge the financial support by the Federal State of Bremen in the framework of the ISIS (Integrated Solutions in Sensorial Structure Engineering) Sensorial Materials Scientific Centre (www.isis.uni-bremen.de).

\section{References}

[1] Meltz, G., Morey, W. W., Glenn, W. H., 1989, Formation of Bragg gratings in optical fibers by a transverse holographic method, Optics Letters, 14:823-825.

[2] Hill, K. O., Fujii, Y., Johnson, D. C., Kawasaki, B. S., 1978, Photosensitivity in Optical Fiber Waveguides: Application to Reflection Filter Fabrication, Applied Physics Letters, 32:647-649. [3] Morey, W. W., Meltz, G., Glenn, W. H., eds.: Udd, E., R. P. Depaula, 1989, Fiber optic Bragg grating sensors, Society of PhotoOptical Instrumentation Engineers (SPIE) Conference Series, 1169:98-107.

[4] Meltz, G., Morey, W. W., 1995, Wavelength Shifts in Fiber Bragg Gratings due to Changes in the Cladding Properties. Photosensitivity and Quadratic Nonlinearity in Glass Waveguides: Fundamentals and Applications, OSA Technical Digest Series (Optical Society of America, Washington, D.C.), 22:225-228.

[5] Xu, M.; Archambault, J.; Reekie, L. \& Dakin, J., 1994, Thermally-compensated bending gauge using surface-mounted fibre gratings, International Journal of Optoelectronics, London; New York: Taylor \& Francis, 9:281-284.

[6] Gander, M.; MacPherson, W.; McBride, R.; Jones, J.; Zhang, L.; Bennion, I.; Blanchard, P.; Burnett, J. \& Greenaway, A., 2000, Bend measurement using Bragg gratings in multicore fibre, Electronics Letters, 36:120-121.

[7] Lefevre, H., 1980, Single-mode fibre fractional wave devices and polarisation controllers, Electronics Letters, 16:778 -780. 